\title{
Grand challenges of brain computer interfaces in the years to come
}

\author{
Eilon Vaadia ${ }^{1 *}$ and Niels Birbaumer ${ }^{2,3 * *}$ \\ Interdisciplinary Center for Neural Computation and the Edmond and Lily Safra Center for Brain Sciences, The Hebrew University, Jerusalem, Israel \\ 2 Istituto di Ricovero e Cura a Carattere Scientifico, Ospedale San Camillo, Italy \\ 3 Institute of Medical Psychology and Behavioral Neurobiology, Eberhard-Karls-University, Tübingen, Germany \\ *Correspondence: *eilonv@ekmd.huji.ac.il; **niels.birbaumer@uni-tuebingen.de
}

Invasive and non-invasive brain machine interface research is a fast growing field, but a series of important challenges will have to be met to bring this to a level that will significantly impact patients. An increasing number of neurologists, neurosurgeon, neuroscientists, theoreticians and computer engineers have become interested in the field, and their work gives hope for significant breakthroughs. The first challenge we face is in the realm of socio-economics. It is essential to have a worldwide network of collaborations and information exchange between all disciplines, including allocation of much larger resources for the task. Mankind needs to learn how to combine the natural tendencies of individuals for personal achievements with the fact that we humans are social animals that made the best by synergistic social interactions and associations to larger teams, tribes and nations. The challenge is to create a worldwide feeling of a united mission. In this sense, we should adopt the brain strategy; none of us humans feel like "a bunch of neurons". Yet, our brains, which make us what we are, are a collection of billions of cells that "know" how to interact and generate intelligence, emotions and creativity. This is in fact a great challenge for the world population in all aspects of science and society.

The major scientific challenge may sound naive and obvious and yet, it is still in debate. How advanced will our understanding of the brain have to be before we can understand its intentions from reading its electrical activity? At present there are views like "using the brain we can not understand the brain", or "the brain is too complex to understand." In fact, the brain's complexity may reside only in our thinking. We have lots of data about the brain, but no single person knows it all. We have different ideas and theories, but no true testable global theory about the brain.

Without such theory, we keep "measuring things." Consider for example, a non-invasive technique like recordings of the EEG; imagine that you try to understand a lecture given by millions of people, while each of them delivers (at the same time) a small fraction of the lecture, and all you hear are the mixed, blurred, echoed voices. Alternatively, consider the invasive techniques like recordings of single neuron activity (or even simultaneous recordings of say 500 neurons). Here, we try to understand a supercomputer with millions and billions of interacting integrated circuits, by recording currents from a very small sample of these circuits, not even knowing the accurate connectivity, while our measuring devices shortcut other parts.

The grand challenges for BMI research are intimately linked to these challenges in brain science in general. The most important aspect of BMIs, especially neuroprosthetics, is its aspiration to achieve better clinical applications. While many clinical treatments have been used even before the system is fully understood, it is intuitively clear that once we fully understand how the device works, it becomes easier to fix it. This is why we take our car or TV to be fixed by experts rather than try to move around wires and see if it works. This is why the experts at the garage or electronics shop must understand the mechanism of the devices and use good tools in order to detect problems and fix them.

Going top-down into the practical challenges of translational and clinical research towards useful brain machine interfaces, we can observe dramatic developments along with difficult problems. As more and more groups are involved in this scientific endeavor, the future looks demanding and promising at the same time. Above, we discussed the origin of the two major challenges (1) Theory: to get the meaning of signals measured (good theory of how the brain works). If one wishes to reveal, for example, if the brain intends to move an arm, at the very least one must predict the brain activity expected for each movement. For more general predictions, we would need a deeper and more global theory. (2) Data acquisition and interpretation: To better listen to the brain, we need good ears and better systems that know how to listen.

The first steps in theories, regarding the principles of brain function that are most relevant to neuroprosthetics, come from computational sensorimotor control (Kawato, 1999; Shadmehr and Krakauer, 2008; Todorov, 2004; Wolpert and Ghahramani 2000). In a recent review, Lalazar and Vaadia presented the wider view suggesting that all brain functions are not based on a serial machine that reads sensory inputs and respond to them; rather, the brain is a memory based prediction machine in which experiences of relations between actions and their results build in the brain internal models. In the case of sensorimotor associations, these models predict the expected sensory inputs and the results of its own actions, and bring about perception. Thus - what we perceive is not necessarily the world as it is, but the assessment of "brain reality" (Lalazar and Vaadia, 2008). In the words of Noe (giving the example of visual perception) "The experience of seeing occurs when the organism masters what we call the governing laws of sensorimotor contingency" (Noë, 2005). This is a debatable approach that can still be adopted when we try to construct a machine that interprets brain activity. Naturally, the challenge is to test such theory and pursue other theories.

The second problem is our relatively poor ability to extract the relevant information from the monitored brain activity. At present we use various methods to monitor brain activity at different levels, from highly invasive to non-invasive ones. The activity, in all cases, provides only partial and "noisy" information about the 
subject's intentions. Moreover, the activity changes continuously, either due to technical problems such as unstable recordings or due to the inherent adaptive nature of the brain itself, which modifies its activity to the subject's experience. Furthermore, the coding scheme by which the brain actually uses to encode information is still highly debated. Approaches to address this challenge are demonstrated in several publications of recent years (for example, Braun et al., 2005; Kim et al., 2006; Krauledat et al., 2008; Wu and Hatsopoulos, 2008; Wu et al., 2006). While facing this challenge, one has to keep in mind the dynamics of behavior and the predictive nature of the sensorimotor internal models. Consequently, we must learn the relevant dynamics of neural processing. It is therefore essential to improve our understanding of the adaptive nature of the brain. Interestingly, we find that this may be an easier task than we might think, since the brain is quite good at this task. Cortical maps are highly dynamic, even at adulthood (Buonomano and Merzenich, 1998), and firing rates of single cells as well as neuronal interactions modify quite rapidly during sensorimotor learning (Gandolfo et al., 2000; Jarosiewicz et al., 2008; Laubach et al., 2000; Mitz et al., 1991; Paz and Vaadia, 2009; Paz et al., 2003; Zach et al., 2008).

To facilitate the development of brain-driven artificial devices that produce natural-like movements, this line of studies should be continued, with special emphasis on developing optimal learning schemes, adapted to the constraints of human motor learning and performance under variable conditions and using classical and instrumental conditioning to teach the brain how to interact with the machine. One line of research in Vaadia lab uses the theory of sensorimotor predictive loops and implements it in showing that BMI is dramatically improved by adopting this principle. The algorithm is not only monitoring the brain activity, but also adapting continuously in the background to its changes, while it controls behavior at the same time. Using this principle, monkeys and machines learned to "work together" in tens of seconds even when the model is started from scratch during every recording day. Thus, these results suggest that even totally paralyzed patients will be able to train themselves and the computer quite rapidly (in 1-2 min) even if the brain activity changes from minute to minute and day to day (Shpigelman et al., 2009).

The idea of adaptation also serves the basic concept of biofeedback which is the basis for the use of neurofeedback in animals and humans. It has already proven successful in human subjects when used to train people to change a particular brain activity through feedback and reward (instrumental learning). For both types of strategies, some proof-of-principle demonstrations of their clinical effectiveness exist but lack larger controlled trials. Neurofeedback of slow cortical potentials and sensorimotor EEGrhythm (SMR) has produced improvements of attention and school performance in children with attention deficit disorder and hyperactivity (ADHD) compared to different control conditions such as placebo training and stimulant medication (Strehl et al., 2006). In drug resistant focal epilepsy, not only were substantial reduction in seizures reported, but also large gains in IQ and cognitive functioning were also demonstrated (Kotchoubey et al., 2001; Strehl et al., 2005) after training of slow cortical potential control, indicating that neurofeedback is a promising tool to improve cognitive functioning in some brain disorders.
The situation is similar in clinical brain-computer-interface research. While animal experiments using implanted microelectrodes in non-human primates have demonstrated perfect brain control of artificial hands or paralyzed limbs from ensembles of firing neurons in motor cortices after training, only one study with eight chronic stroke patients without residual movement capacity using a non-invasive magnetoencephalographically controlled prosthetic hand $\mathrm{BCI}$ is available. Most patients were able to open and close their paralyzed hands fixed on the orthosis with sensorimotor oscillations from their motor cortices; however, no gains in motor control outside the laboratory were reported (Buch et al., 2008).

For paralyzed spinal cord patients, a few single case demonstrations are available (Pfurtscheller et al., 2005). EEG or MEG have to be combined with intelligent peripheries and robots; in motor control only four-dimensions of control are possible (i.e., right-left, front-back). Even with sophisticated algorithms, EEG cannot provide better classification solutions due to its biophysical limitations (Birbaumer et al., 1990). For most applications, however, EEG may remain the $\mathrm{BCI}$ of choice.

Verbal communication with completely paralyzed locked-in patients mostly suffering from amyotrophic lateral sclerosis (ALS) with non-invasive BCIs using different brain signals from the EEG for selecting letters or "yes" and "no" answers in a computer menu was described in several reports (Birbaumer et al., 2008). However, in completely-locked-in patients without any remaining eye-movement control, BCI training was not successful.

Future BCIs for direct brain communication should rely on strategies requiring no or minimal cognitive-attentional effort and use mainly implicit learning. Locked-in, vegetative state (VS) and advanced Alzheimer patients should be trained to produce reflexive or automatic brain responses to questions or cues which can then be used as affirmative answers or rejections. Implantation of electrodes epidurally will improve signal to noise ratio and help the patient to regulate his/her Electrocorticogram (ECoG).

Classical conditioning of brain potentials and oscillations using clearly differentiable conditioned and unconditioned auditory or somatosensory stimuli (vision is often compromised) may overcome the problem of voluntary, effortful conscious processing that is not possible in these patient groups.

In chronic stroke, spinal cord injury and other forms of motor paralysis, a recent demonstration in reversibly paralyzed monkeys by Moritz et al. (2008) should be translated into human application. Here, the monkey was trained to produce spike sequences with operant conditioning from a few cells in the motor cortex to activate functional electric stimulation (FES) electrodes fixed to the paralyzed fingers. Invasive BMIs using implanted micro-or macroelectrodes in human patients need to be tested experimentally as tested with non-invasive EEG/MEG, near-infrared-spectroscopy and magnetic resonance imaging BCIs. Most paralyzed patients refuse neurosurgical procedures as they are too risky; even if less flexible and error-prone, non-invasive measures will complement invasive BCIs. In neurofeedback the situation is less complicated because some first controlled demonstrations are already available and only large controlled trials are missing.

Yet, there is a lot to improve in our ability to read brain activity. The noninvasive studies, thus far suffer several problems; at present 
some have low spatial resolution (including EEG and fMRI) and low temporal resolution (fMRI). In addition many of the devices are not always practical for daily use. Likewise, invasive technologies are not too useful for clinical applications at the current state. At present one can implant micro-arrays of many electrodes, but most are still damaging the tissue to some extent and do not last for many years. One example of efforts in the right direction are described by Kennedy and colleagues who developed Neurotrophic electrodes (Bartels et al., 2008). Telemetry techniques are still limited and do not allow transmission of full wave signals or even just the action potentials from hundreds of electrodes at sufficient speed. Yet, an example of the right steps were recently made by developing a 96-channel implantable data acquisition system that performs spike detection and extraction and wirelessly transmits data to an external unit (Rizk et al., 2009). Some reader may argue that we are almost there. Yet, most scientists believe that it takes better technologies to get to the desired devices that will provide samples of large number of single neurons using telemetry and stable recordings, for many years and with no damage to the brain tissue. One important light at the end of the tunnel may be provided in the future by the subfield of nanotechnologies, which will develop nano-detectors which may be implanted inertly in the brain and measure local electrical activity. When that day comes, we will able to implant thousands of inert detectors that can transmit the compressed version of the information outside of the brain - it will represent a significant revolution in the field of BMI. Likewise, the technological challenge for using noninvasive techniques involves increasing of spatial and temporal resolution and miniaturization of the devices. This poses engineering challenges that may look sometime trivial, yet important like for example, miniaturization of power supply to the some electronic components that must be implanted. These developments will be used not only for motor prosthesis but also other treatments like closed loop deep brain stimulation. This must be improved to include recording of brain activity, which would allow for dynamic, adaptive stimulation that will condition the brain activity to restore normal activity when it goes astray (like in Parkinson's disease).

Finally, while all these smart detectors and algorithms will be interfaced to the brain on one side, one can't forget or neglect the other side: interfacing the output of these devices to effectors. The ultimate solution in neuroprosthetics will be control of the natural limb; an intermediate solution may range from controlling a computer or robotic devices (arm, wheel-chair, hand etc).

Furthermore, the long-term challenge may bring this field to much broader clinical applications, in improvements of not only paralysis but also other brain functions. This would include cognitive function and psychiatric conditions, like psychopathy, obsessive compulsive disorder, depression and schizophrenia that have been already attempted using deep brain stimulation and behavioral treatments, but require extensive work to achieve finetuned, closed-loop recording-stimulation that will allow conditioning of brain activity to switch from the disease patterns of electrical activity to normal patterns. First reports of operant conditioning of subcortical and cortical nuclei with real-time functional magnetic resonance imaging (rt-fMRI-BCI) are promising in this respect (Weiskopf et al., 2007). Bearing in mind the theory that the brain deals with coordinating its internal models with the incoming inputs and results of its actions, it is clear that these tasks are not impossible. Expansion of clinical use will bring about serious ethical issues, which will pose yet another grand challenge to mankind, and scientists must push this ethical challenge aside.

\section{REFERENCES}

Bartels, J., Andreasen, D., Ehirim, P., Mao, H., Seibert, S., Wright, E. J., and Kennedy, P. (2008). Neurotrophic electrode: method of assembly and implantation into human motor speech cortex. J. Neurosci. Methods 174, 168-176.

Birbaumer, N., Elbert, T., Canavan, A., and Rockstroh, B. (1990). Slow potentials of cerebral cortex and behavior. Physiol. Rev. 70, 1-41.

Birbaumer, N., Ramos Murguialday, A., and Cohen, L. (2008). BrainComputer-Interface (BCI) in paralysis. Curr. Opin. Neurol. 21, 634-638.

Braun, D.A.,Aertsen,A., Paz, R., Vaadia, E., Rotter, S., and Mehring, C. (2005). Interpreting Sensorimotor Learning by Adaptive Optimal Control Program No. 822.4. 2005. Washington, DC, Society for Neuroscience.

Buch, E., Weber, C., Cohen, L. G., Braun, C., Dimyan, M., Ard, T., Mellinger, J., Caria, A., Soekadar, S., Fourkas, A., and Birbaumer, N. (2008). Think to move: a neuromagnetic braincomputer interface (BCI) system for chronic stroke. Stroke 39, 910-917.
Buonomano, D. V., and Merzenich, M. M. (1998). Cortical plasticity: from synapses to maps. Annu. Rev. Neurosci. 21, 149-186. Review.

Gandolfo, F., Li, C., Benda, B. J., Schioppa, C. P., and Bizzi, E. (2000). Cortical correlates of learning in monkeys adapting to a new dynamical environment. Proc. Natl. Acad. Sci. U.S.A. 97, 2259-2263.

Jarosiewicz, B., Chase, S. M., Fraser, G. W. Velliste, M., Kass, R. E., and Schwartz, A. B. (2008). Functional network reorganization during learning in a brain-computer interface paradigm. Proc. Natl. Acad. Sci. U.S.A. 105, 19486-19491.

Kawato, M. (1999). Internal models for motor control and trajectory planning. Curr. Opin. Neurobiol. 9, 718-727.

Kim, S. P., Sanchez, J. C., Rao, Y. N., Erdogmus, D., Carmena, J. M., Lebedev, M. A., Nicolelis, M. A., and Principe, J. C. (2006). A comparison of optimal MIMO linear and nonlinear models for brain-machine interfaces. J. Neural Eng. 3, 145-161.
Kotchoubey, B., Strehl, U., Uhlmann, C. Holzapfel, S., König, M., Fröscher, W. Blankenhorn, V., and Birbaumer, N. (2001). Modification of slow cortical potentials in patients with refractory epilepsy: a controlled outcome study. Epilepsia 42, 406-416.

Krauledat, M., Tangermann, M., Blankertz, B., and Muller, K. R.(2008) Towards zero training for braincomputer interfacing. PLOS ONE 3 e2967.

Lalazar, H., and Vaadia, E. (2008). Neural basis of sensorimotor learning: modifying internal models. Curr. Opin. Neurobiol. 18, 573-581.

Laubach, M., Wessberg, J., and Nicolelis, M. A. (2000). Cortical ensemble activity increasingly predicts behaviour outcomes during learning of a motor task Nature 405, 567-571.

Mitz, A. R., Godschalk, M., and Wise, S. P. (1991).Learning-dependent neuronal activity in the premotor cortex: activity during the acquisition of conditional motor associations. J. Neurosci. 11, 1855-1872.
Moritz, C. T., Perlmutter, S. I., and Fetz, E. E. (2008). Direct control of paralysed muscles by cortical neurons. Nature 456, 639-642.

Noë, A. (2005). Action in Perception. Cambridge, MIT.

Paz, R., Boraud, T., Natan, C., Bergman, H., and Vaadia, E. (2003). Preparatory activity in motor cortex reflects learning of local visuomotor skills. Nat. Neurosci. 6, 882-890.

Paz, R., and Vaadia, E. (2009). Learning from learning: what can visuomotor adaptations tell us about the neuronal representation of movement? Adv. Exp. Med. Biol. 629, 221-242.

Pfurtscheller, G., Neuper, C., and Birbaumer, N. (2005). Human BrainComputer Interface (BCI). In Motor Cortex in Voluntary Movements. A Distributed System for Distributed Functions, Alexa Riehle and Eilon Vaadia, eds (Boca Raton, CRC Press), pp. 367-401.

Rizk, M., Bossetti, C. A., Jochum, T. A., Callender, S. H., Nicolelis, M. A., Turner, D. A., and Wolf, P. D. (2009). 
A fully implantable 96-channel neural data acquisition system. J. Neural Eng. 6, 026002 .

Shadmehr, R., and Krakauer, J. W. (2008). A computational neuroanatomy for motor control. Exp. Brain Res. 185, 359-381.

Shpigelman, L., Lalazar, H., and Vaadia, E. (2009). Kernel-ARMA for Hand Tracking and Brain-Machine Interfacing During 3D Motor Control Advances in Neural Information Processing Systems (NIPS) 21. MIT Press, Cambridge.

Strehl, U., Kotchoubey, B., Trevorrow, T. and Birbaumer, N. (2005). Predictors of seizure reduction after selfregulation of slow cortical potentials as a treatment of drug-resistant epilepsy. Epilepsy Behav. 6, 156-166.

Strehl, U., Leins, U., Goth, G., Klinger, C., Hinterberger, T., and Birbaumer, N. (2006).Selfregulation of slow cortical potentials - a new treatment for children with attention-deficit/hyperactivity disorder. Pediatrics 118, 1530-1540.

Todorov, E. (2004). Optimality principles in sensorimotor control. Nat. Neurosci. 7, 907-915.

Weiskopf, N., Sitaram, R., Josephs, O., Veit, R., Scharnowski, F., Goebel, R., Birbaumer, N., Deichmann, R., and Mathiak, K. (2007). Realtime functional magnetic resonance imaging: methods and applications. Magn. Reson. Imaging 25, 989-1003.

Wolpert, D. M., and Ghahramani, Z. (2000). Computational principles of movement neuroscience. Nat. Neurosci. 3(Suppl), 1212-1217.

Wu, W., Gao, Y., Bienenstock, E., Donoghue, J.P., and Black, M. J. (2006). Bayesian population decoding of motor cortical activity using a Kalman filter. Neural Comput. 18, 80-118.

Wu, W., and Hatsopoulos, N. G. (2008). Real-time decoding of nonstationary neural activity in motor cortex. IEEE Trans. Neural Syst. Rehabil. Eng. 16, 213-222.

Zach, N., Inbar, D., Grinvald, Y. Bergman, H., and Vaadia, E. (2008).
Emergence of novel representations in primary motor cortex and premotor neurons during associative learning. J. Neurosci. 28, 9545-9556.

Received: 19 May 2009; published: 15 September 2009

Citation: Front. Neurosci. (2009) 3, 2: 151 154. doi: 10.3389/neuro.01.015.2009 Copyright (c) 2009 Vaadia and Birbaumer. This is an open-access publication subject to an exclusive license agreement between the authors and the Frontiers Research Foundation, which permits unrestricted use, distribution, and reproduction in any medium, provided the original authors and source are credited.

\title{
Herding cats: the sociology of data integration
}

\author{
Robert W. Williams ${ }^{1 *}$ \\ ' Department of Anatomy and Neurobiology, Center for Integrative and Translational Genomics, University of Tennessee Health Science Center, Memphis, TN, USA \\ *Correspondence: rwilliam@nb.utmem.edu
}

Upon this gifted age, in its dark hour, Rains from the sky a meteoric shower Of facts... they lie unquestioned, uncombined. Wisdom enough to leech of our ill Is daily spun; but there exists no loom To weave it into fabric;

—Edna St. Vincent Millay (from Huntsman, What Quarry? 1939)

\section{THE CHALLENGE OF PREDICTION}

The age of personalized medicine and genomics is upon us and we are facing a grand challenge - or a brick wall. Once we have finally gained a near complete compendium of fundamental mechanisms, connections, and developmental sequences - an encyclopedia of biology, bodies, brains, and behavior - can we achieve the data density and integration needed to develop holistic and robust models that generate useful predictions? Will we be able to distinguish between personalized genomics and a horoscope? What new types of resources, data sets, and synthetic frameworks are needed to make correct prognoses and recommend actions? What is my personal risk for Alzheimer's disease, and what should I do about it today?

The complexity of biological systems implies that a parts list of mechanisms and processes, however complete, will not be up to the task of making good predictions. We need a way to test drive our models using a system that has the same level of complexity as human populations. I will describe an effective approach that relies on genetic reference panels (GRPs) that can be used to make and test predictions from base pair to behavior. I will describe how scientists can retain their independence while explicitly contributing to a fabric of tightly woven quantitative data.

\section{THE COLLECTIVE COST OF SCIENTIFIC INDEPENDENCE}

Scientists are trained to think independently and critically. It is inevitable that we like to do things our own way, generating and using data from experiments we designed ourselves. This approach is not a self-indulgent luxury - it is an essential attribute of innovative science, enshrined in the ways we evaluate and fund new and ongoing research. Independence contributes to the stirring cacophony of competing ideas that moves us toward a deeper understanding of biological processes.

Yet, independence has a cost. The scope of studies from single groups is limited by their technical and analytic proficiency and by modest budgets. The collective result is a fragmented, half-hidden literature and a fragmentary and rapidly evaporating collection of raw data, generated using different species and strains raised under different conditions, treated using varied paradigms, and measured using different equipment. Of course the pieces do not fit together! They were never intended to fit into any unified design. It is no surprise that integration of data sets and of key results is difficult; sometimes impossible. Ronald Fisher pointed out that "a competent overhauling of the process of collection, or of experimental design, may often increase the yield (precision of results) ten or twelve fold, for the same cost in time and labour" (Rao, 1992). Fisher meant this in the context of a single 